\title{
Adaptive Illumination Source for Multispectral Vision System Applied to Material Discrimination
}

\author{
Olga M. Conde, Adolfo Cobo, Paulino Cantero, David Conde, Jesús Mirapeix, \\ Ana M. Cubillas, José M. López-Higuera \\ Photonics Engineering Group, Dep. TEISA, Universidad de Cantabria, Avda. Los Castros s/n, 39005 \\ Santander, Spain
}

\begin{abstract}
A multispectral system based on a monochrome camera and an adaptive illumination source is presented in this paper. Its preliminary application is focused on material discrimination for food and beverage industries, where monochrome, color and infrared imaging have been successfully applied for this task. This work proposes a different approach, in which the relevant wavelengths for the required discrimination task are selected in advance using a Sequential Forward Floating Selection (SFFS) Algorithm. A light source, based on Light Emitting Diodes (LEDs) at these wavelengths is then used to sequentially illuminate the material under analysis, and the resulting images are captured by a CCD camera with spectral response in the entire range of the selected wavelengths. Finally, the several multispectral planes obtained are processed using a Spectral Angle Mapping (SAM) algorithm, whose output is the desired material classification. Among other advantages, this approach of controlled and specific illumination produces multispectral imaging with a simple monochrome camera, and cold illumination restricted to specific relevant wavelengths, which is desirable for the food and beverage industry. The proposed system has been tested with success for the automatic detection of foreign object in the tobacco processing industry.
\end{abstract}

Keywords: Multispectral system, high-bright LEDs, imaging spectroscopy, automatic discrimination, material characterization.

\section{INTRODUCTION}

Automated machine vision has developed over the years in many areas, one of them being the range of wavelengths used for inspection and also the number of spectral ranges that are individually processed to obtain the desired information. The change from a single monochrome wide spectral band to color imaging (three bands, RGB, in the visible range) has result in better performance in typical discrimination tasks. Ultimately, this concept has been extended to more spectral bands and to wavelengths outside the visible range, resulting in more capable imaging systems, commonly known as multispectral imaging systems [1][2]. A typical multispectral system comprises a white light source to illuminate the material under study, and an imaging device which produce 2D images planes at different wavelengths. The wavelength resolution is usually provided by optical filters or diffraction elements [3], and it can be very high, with hundred or thousand of resolved spectral bands. This result in complex and expensive instruments with a large amount of data to be processed. One interesting option is to restrict the spatial resolution to one-dimension, a line, so a single 2D plane contains both spatial and spectral resolution. This approach allows a simpler instrument based on a conventional imaging camera and the proper diffractive optics [4].

For all of those systems based on spectroscopic analysis, data processing required to perform the discrimination task involved two steps: dimensional reduction and the discrimination itself. The first step is important when the spectral resolution is higher than needed, as several works have demonstrated that in most applications, only a few discrete spectral bands contain enough information to perform the discrimination with acceptable error, thus reducing the computational cost of the subsequent steps and of the entire process [5]. To perform adequate dimensional reduction actions, samples of the different materials to be discriminated should be available in order to identify their relevant features. One approach to perform the dimensional reduction is the Principal Components Analysis (PCA) [6] which performs data compression maintaining the relevant information contained in second-order statistics of the data. The spectrum of each spatial point is projected over a vector basis different from the spectral wavelengths, and related with

Optical and Digital Image Processing, edited by Peter Schelkens, Touradj Ebrahimi, Gabriel Cristóbal, Frédéric Truchetet, Proc. of SPIE Vol. 7000, 70000Z, (2008) · 0277-786X/08/\$18 · doi: 10.1117/12.781496 
the directions where their variance deviate the most. This projection is widely known and employed but does not provide a physical insight of the problem. However, this objective can be achieved in a different way that consists in identifying and discarding those spectral bands, or features, not contributing to the classification task. After that, the selected and maintained spectral bands are employed by the subsequent classification algorithm in the same way as principal components. These redundancy removal algorithms are called feature selection methods, and they come to solve on disadvantages of feature extraction techniques, such as PCA, where the results cannot be associated to spectral bands with physical meaning [7]. One of these feature selection algorithms is the Sequential Floating Forward Selection algorithm (SFFS), that has been successfully applied to reduce the dimensionality, or the features, of hyperspectral data prior to the discrimination step [3], [7]-[9].

For the discrimination or classification task, several approaches have been proposed. Among others, Artificial Neural Networks (ANN) [10] are becoming more popular for pattern recognition due to their advantages for facing with nonlinearity and are also beginning to play an increasingly significant place in the food industry [11][12]. Another simple classification approach is the Spectral Angle Mapping (SAM) [13][14]. This algorithm determines the similarity between two spectra by calculating the "spectral angle" between them. Spectra are considered as vectors in a space with dimensionality equal to the number of preserved bands. Small angles between the two spectrums indicate high similarity and high angles indicate low similarity. Since SAM uses only the direction of the spectra and not their length, the method is insensitive to the variations of gain factor of the measurement, the diffuse reflectance of the material in this case.

One possible optimization of the above mentioned multispectral imaging systems and algorithms is to perform the dimensional reduction inversely or in advance, thus avoiding the capturing and elimination of redundant or nonsignificant wavelengths. As most of the discrimination tasks can be performed with only a few discrete spectral bands, this can improve the efficiency and reduce the complexity of the system. Additionally, most of the energy emitted by the white light source is therefore not useful for the discrimination task, as only a few of the all emitted wavelengths are analyzed. If the light source emission were restricted to the significant wavelengths, also the excess heat produced by conventional white light sources, that is a major concern in some applications for the food and beverage industries, could be reduced.

In this paper, we propose a light source with discrete wavelengths, whose values are selected in advance using the SFFS algorithm for a particular discrimination problem. The light source is based on multiple high-bright LEDs, a set of them for every wavelength, that are sequentially activated by a computer. For each wavelength, an image is captured and stored by a conventional monochrome Silicon-based CCD camera, to obtain multiple spectral planes of the material. A discrimination algorithm (SAM) is then applied to the images to obtain a classification of the different materials appearing in the image, based on their spectral features.

This system has been applied with success to one particular problem: the detection and classification of foreign objects that can be mixed with chopped tobacco leaves in the tobacco processing industry. The next section is focused in the wavelength selection for this particular application, section 3 give details of the adaptive light source, the experimental setup and results are described in detail in section 4, while section 5 deals with the main conclusions from the results and future works, some of them ongoing at the moment, to improve the proposed system.

\section{WAVELENGTHS SELECTION}

A key parameter of the proposed system is the definition of the set of discrete wavelengths selected to sequentially illuminate the surface under inspection. This set of wavelengths is specific for each discrimination problem, as it depends strongly in the diffuse reflectance characteristics of the surface of the different materials that need to be classified. The proposed algorithm to obtain these significant wavelengths is the Sequential Floating Forward Selection (SFFS) [3][7]. The aim of SFFS is to select $\mathrm{M}$ out of the $\mathrm{N}$ initial spectral bands, where $\mathrm{M}<\mathrm{N}$. Obviously, this reduction would increase the classification error, unless those $\mathrm{M}$ spectral bands that best discriminate among the classes were selected. Then, SFFS assumes that there are several classes, or statistical distributions, to be discriminated therefore it is based on class separability. Classification performance is an alternative selection criterion. While an algorithm based on classification performance selects those features that provide higher classification accuracy, an algorithm based on class separability chooses those spectral bands maximizing the distance among the classes. On the other hand, the major drawback of the selection based on classification performance resides on its computational performance. It is necessary to train and test

the classifier for every selected feature subset. In addition, the obtained results cannot be extended to other classifiers. 
Feature selection algorithms based on class separability are less time-consuming and independent of the classifier. Separability measurement can be calculated in three different ways [7]:

- By measuring the probabilistic distance between the probability density functions. Typical probabilistic distances are Chernoff, Bhattacharyya and Patrick-Fisher.

- Computation of between-class and within-class scatter matrixes.

- Theoretical measurements of information and correlation.

As in [7], the Bhattacharya distance is here selected as the criterion to measure class separability, it is given by:

$$
J_{B}=\frac{1}{4}\left(\mu_{2}-\mu_{1}\right)^{T}\left[\Sigma_{1}+\Sigma_{2}\right]^{-1}\left(\mu_{2}-\mu_{1}\right)+\frac{1}{2} \ln \left(\frac{\left|\Sigma_{1}+\Sigma_{2}\right|}{2\left(\left|\Sigma_{1}\right| \cdot\left|\Sigma_{2}\right|\right)^{\frac{1}{2}}}\right)
$$

where $\mu_{\mathrm{i}}$ and $\Sigma_{\mathrm{i}}$ are the mean and the covariance matrix for class $i$, where Gaussian distribution of the classes is assumed.

Apart from the selection criterion, an optimal search strategy of the feature bands must be followed because, even if the number of final selected features is known, the evaluation of the performance of each selected subset is unapproachable due to time consumption. SFFS is named "forward" because it begins with one feature and continues adding features. It is also named "floating" because after each forward step (addition of a new spectral band $x_{i n c}$ ) a number of backward steps (removal of several spectral bands $x_{e x c}$ ) are applied as long as the resulting subsets are better than the previously evaluated ones according to the selection criterion. This procedure continues until some termination criterion, which is fixed by the user, is met. In this case, it is the maximum number of features $x_{i n c}$ (wavelengths in this case) to be used.

A graphical scheme of this procedure, where $\mathrm{X}$ is the initial feature subset and $\mathrm{Y}$ is the feature subset selected by means of SFFS, is presented in Figure 1.

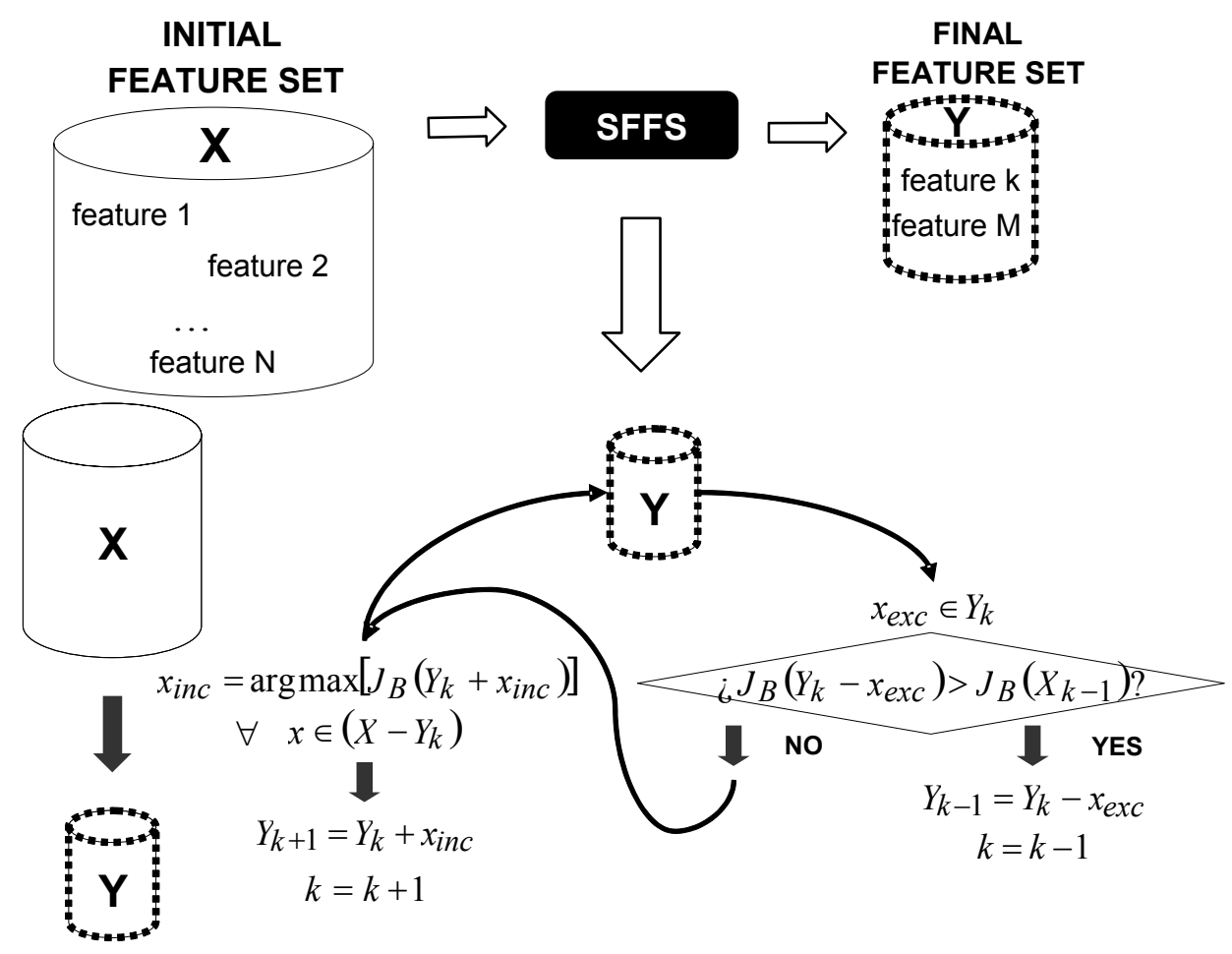

Fig. 1. Representation of the SFFS algorithm for wavelength selection, from X initial spectral bands, to $\mathrm{Y}$ wavelengths. 
For the particular application of foreign objects detection in the tobacco industry, several images of chopped tobacco leaves mixed with other materials have captured with a multispectral set-up under white light illumination [9]. The characterization set-up employs an Imspector ${ }^{\circledR}$ diffractive optics with spectral range from $400 \mathrm{~nm}$ to $100 \mathrm{~nm}$, and a Pixelink ${ }^{\circledR}$ PL-A741 CCD camera with similar spectral range. This setup produces 640 spectral bands along an inspection line of several centimeters long where the material under study is placed. Different materials, considered from the industrial point of view as foreign objects, have been characterized: candy wrappers, lengths of cord, leather and other vegetal dry leaves.

The proposed SFFS algorithm has been applied and the result is shown in Table 1. The selected wavelengths are detailed as a function of the number of light sources to be used. The fewer the number of wavelengths, the larger is the expected discrimination error, but it has been demonstrated in a previous work that with only 6 wavelengths, the error is contained and not much larger than with the original 640 spectral bands [9].

Table 1. Output of the SFFS algorithm: selected wavelengths for several cases of required output band. The expected discrimination error is expected to increase with the decrease of the number of selected wavelengths.

\begin{tabular}{|c|cccccc|}
\hline $\begin{array}{c}\text { NUMBER OF SELECTED } \\
\text { BANDS }\end{array}$ & \multicolumn{5}{|c|}{ SELECTED WAVELENGTHS (nm) } \\
\hline \multirow{2}{*}{12} & 407.5 & 430 & 447.81 & 466.56 & 493.75 & 577.19 \\
& 599.69 & 674.69 & 730.94 & 817.18 & 891.25 & 915.63 \\
\hline \multirow{2}{*}{10} & 407.5 & 430 & 447.81 & 466.56 & 493.75 & 577.19 \\
& 599.69 & 674.69 & 891.25 & 915.63 & & \\
\hline \multirow{2}{*}{8} & 407.5 & 430 & 466.56 & 577.19 & 599.69 & 674.69 \\
& 891.25 & 915.63 & & & & \\
\hline 6 & 407.5 & 430 & 466.56 & 577.19 & 599.69 & 674.69 \\
\hline
\end{tabular}

\section{DESCRIPTION OF THE ADAPTATIVE ILUMINATION SOURCE}

We propose in this paper a light source capable of emitting, sequentially, different discrete wavelengths as a means to obtain different spectral planes of the same image. One key aspect is that the set of wavelengths is obtained in advance by a SFFS algorithm, as described in the previous section. For the problem of foreign material detection and discrimination in the tobacco processing industry, six discrete wavelengths have been selected from a set of 640 initial spectral bands captured by an imaging spectrograph. It is expected that these six wavelengths are the most significant to perform the discrimination task.

For each selected wavelength, a high bright Light Emitting Diode (LED) has been selected, with its central wavelength as close as possible to the required one. The central wavelength, spectral width and optical power are shown in Table 2. The (normalized) experimental spectra of the six LEDs are shown in Figure 2.

Table 2. Parameters of the selected LEDs.

\begin{tabular}{|c|c|c|c|c|}
\hline$\#$ & $\begin{array}{c}\text { Required } \lambda \\
(\mathbf{n m})\end{array}$ & $\begin{array}{c}\text { Central } \lambda \\
(\mathbf{n m})\end{array}$ & $\begin{array}{c}\text { Spectral Width } \\
\text { FWHM(nm) }\end{array}$ & $\begin{array}{c}\text { Optical Power } \\
(\mathbf{m W})\end{array}$ \\
\hline 1 & 407.50 & 405 & 25 & 150 \\
\hline 2 & 430.00 & 430 & 50 & 0,3 \\
\hline 3 & 674.69 & 670 & 25 & 110 \\
\hline 4 & 466.56 & 465 & 20 & 45 \\
\hline 5 & 577.19 & 572 & 25 & 3 \\
\hline 6 & 599.69 & 600 & 15 & 1 \\
\hline
\end{tabular}




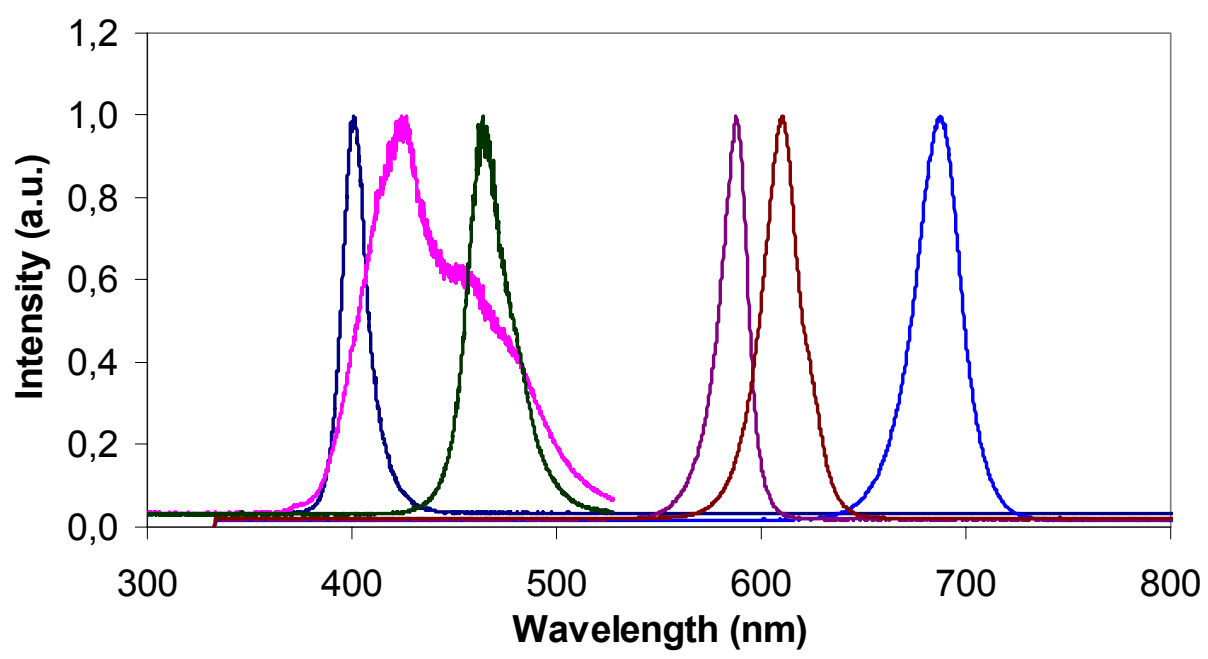

Fig. 2. Experimental spectra of the six LEDs selected for the adaptive light source.

A photograph of a prototype of the light source is shown in Figure 3. The light source includes a microcontroller which is able to switch on and off the different LEDs with controlled current excitation. The operation of this device is controlled by a PC computer by means of a USB interface.

The LEDs has been placed over a Printed Circuit Board (PCB) plane, in a 4x4 array. The placement of each LED source and the number of LEDs for each wavelength depends on the emitted optical power and directivity of the radiation diagram of each one. The objective is to get a uniform illumination over the analysis area, and to balance the emitted power among different wavelengths. The later goal is difficult to reach due to the differences in the emitted power of different LEDs sources, as it is difficult to obtain high bright diodes outside the typical wavelengths (i.e., R, G \&B) used for common illumination purposes. This problem has been resolved by means of a calibration procedure using a reflectance standard, as described in the next lines.

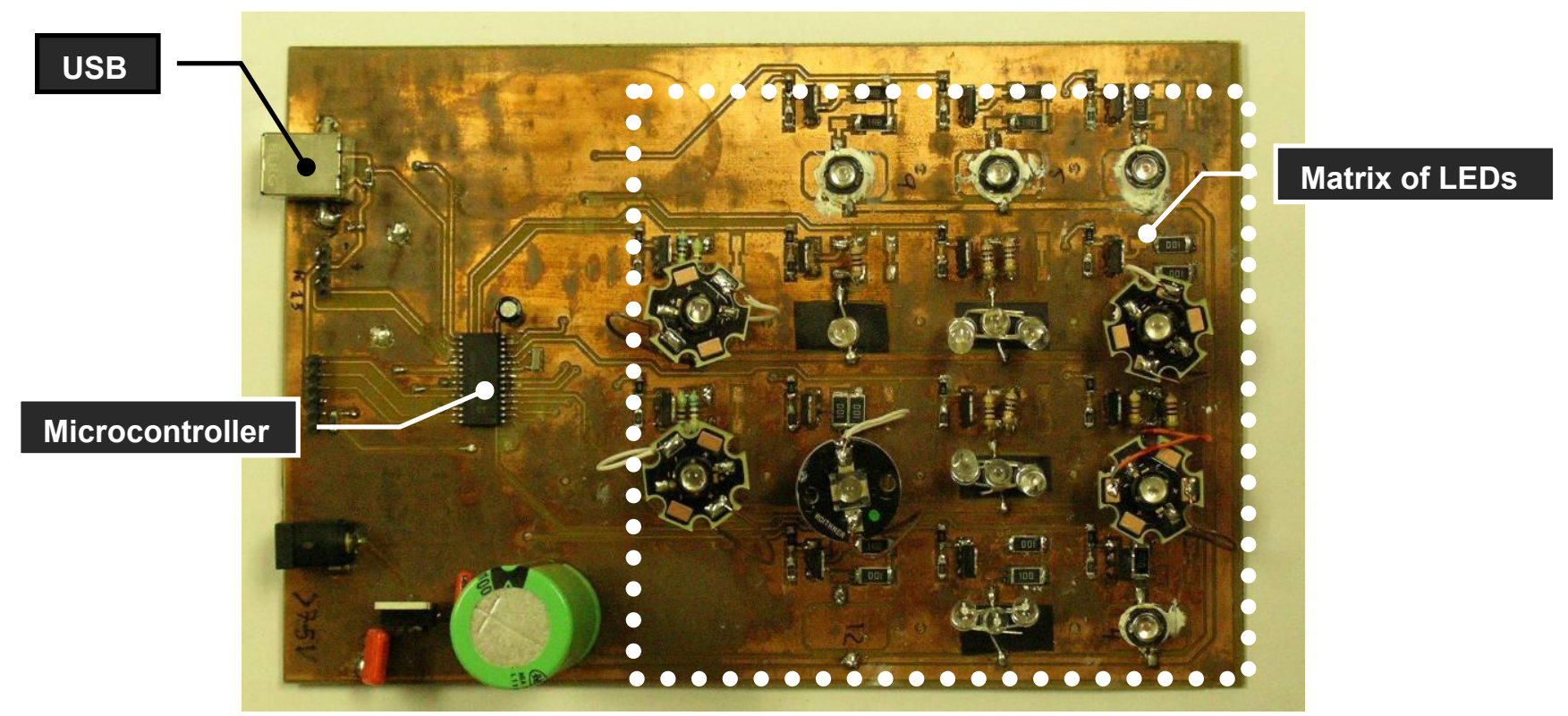

Fig. 3. Photograph of a prototype of the adaptive light source. 


\subsection{Calibration of the light source}

The calibration procedure tries to correct the large differences in optical power of each LEDs source, and the nonuniform spectral response of the optics and the CCD camera. The proposed procedure uses a calibration standard for reflectance measurements, which is based on the Polytetrafluoroethylene (PTFE) material, with flat diffuse reflectance response of $98 \%$ in the range from 250 to $1500 \mathrm{~nm}$.

In the proposed procedure, the camera is aimed at the PTFE surface, and each LED source is switched on in sequence. For each light source, the captured image is analyzed in search of the pixel of highest intensity. Then, the internal gain and the integration time of the CCD camera are modified until the intensity of this pixel is close but lower than the maximum (saturation) value. This step is repeated for each LED source, and the values of gain and integration time are stored. These values are used later during the discrimination task to optimize the capturing parameters for each wavelength.

As an example of the behavior of the proposed light source, it has been used to compose a color image using a monochrome CCD camera. This color image is created by mixing the three monochrome images arising from the selective illumination at the wavelengths of 430,540 and $650 \mathrm{~nm}$, which corresponds to the red, green and blue spectral bands used for color imaging. In Figure 4, the images obtained from a colorful ribbon cable for each wavelength are shown in the upper part, while the image at the bottom of the figure shows the composition of the three planes (color photograph in the original).

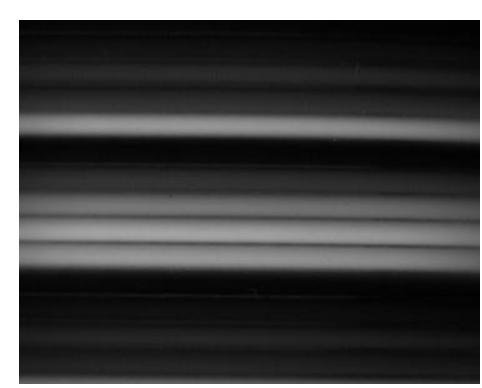

$430 \mathrm{~nm}$

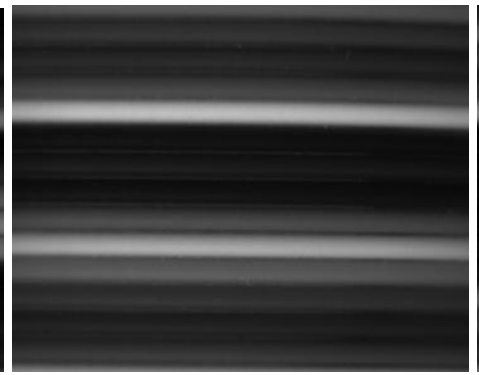

$540 \mathrm{~nm}$

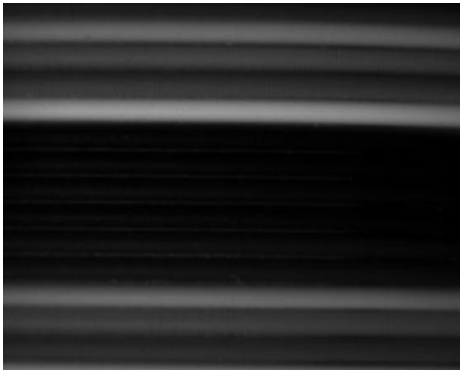

$650 \mathrm{~nm}$

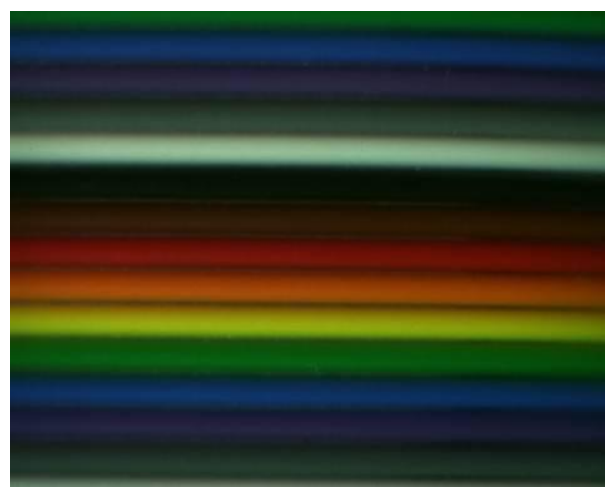

Fig. 4. Top: Imaging of a colorful ribbon cable at three different wavelengths (430, 540 and $650 \mathrm{~nm})$. Composition of the three RGB planes (color photograph in the original).

\section{EXPERIMENTAL RESULTS}

The proposed adaptive illumination system has been applied to the automatic discrimination of foreign objects in a continuous belt carrying chopped tobacco leaves. The early detection of these foreign objects (lengths of cords, candy wrappers, papers, chunks of wood, ...) is an important quality assurance problem in the tobacco processing industry. The 
proposed system has been test in the laboratory for this particular application. The experimental setup is shown in Figure 5 .

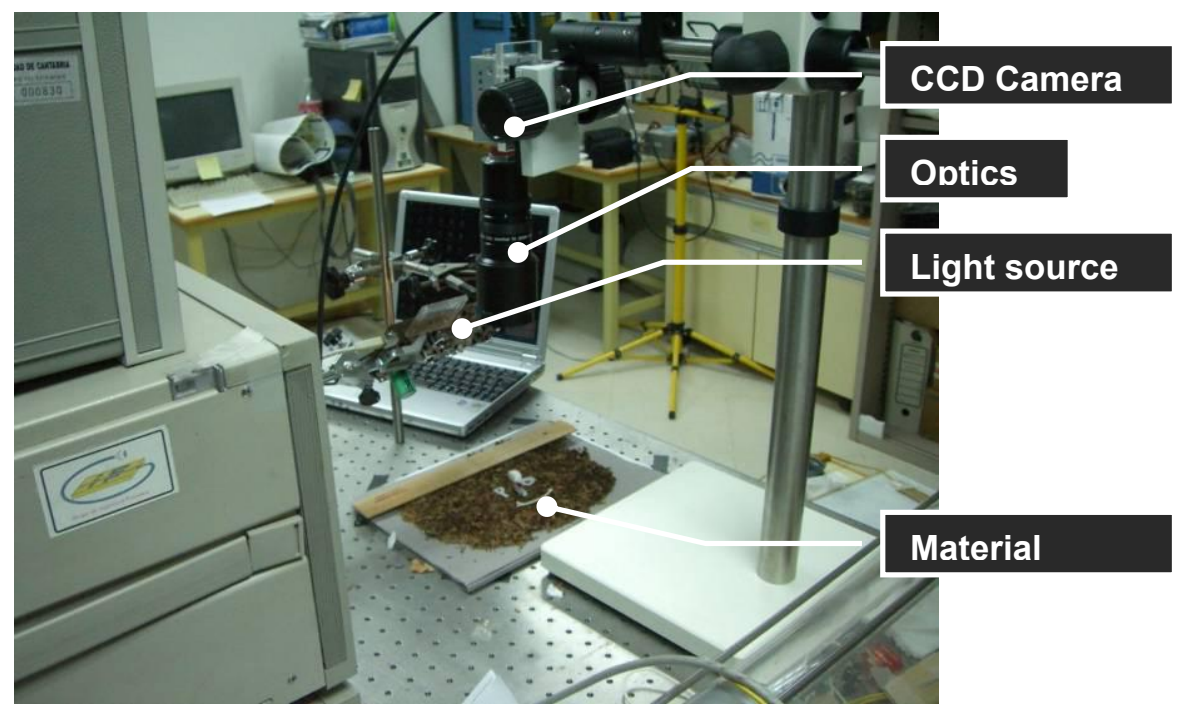

Fig. 5. Experimental setup.

This setup includes the proposed light source (aimed at an angle of about $45^{\circ}$ with respect to the material under test), and a Pixelink PL-A741 CCD monochrome camera equipped with Zoom Lenses (Navitar 7000). The material under study is shown in Figure 6: chopped tobacco leaves of different sizes, a piece of wrapping paper, a piece of leather, a bunch of cotton threads, and two pieces of plastic wrapper.

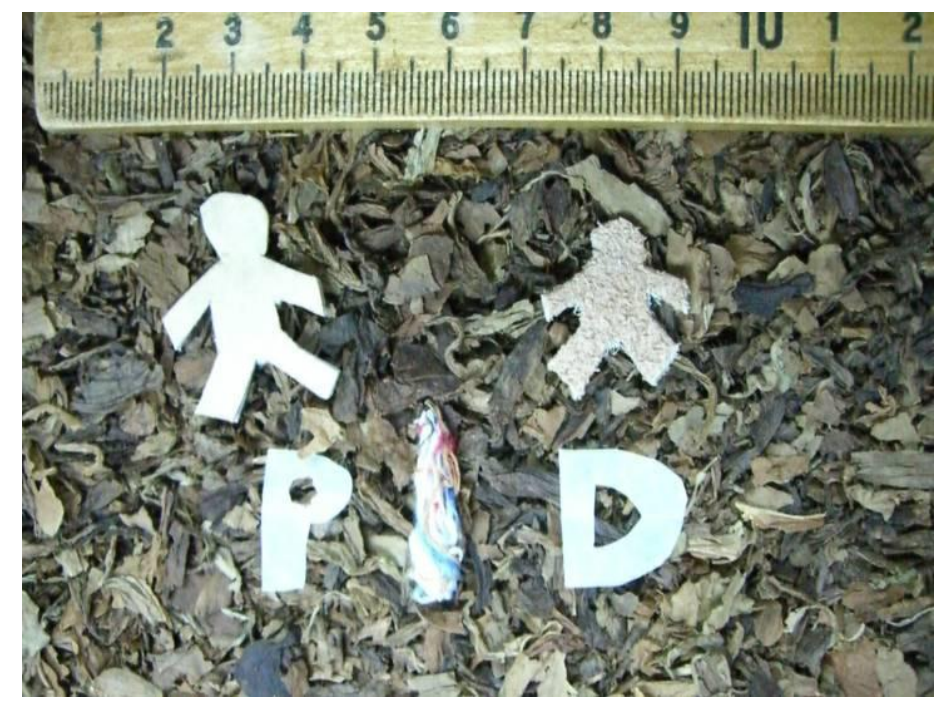

Fig. 6. Material under test: several foreign materials over chopped tobacco leaves.

Six planes at the wavelengths indicated in section 3 have been captured. Each spectral plane has a spatial resolution of $1280 \times 1024$ pixels, corresponding to an area of approximately $12 \times 10 \mathrm{~cm}$, that is, about $0.1 \mathrm{~mm}$ per pixel. The Spectral Angle Mapping (SAM) algorithm [14] described in section 1 has been applied to the multispectral images, with the aim to discriminate between tobacco and non-tobacco materials. The result can be seen in Figure 7. The white areas correspond to pixels classified as tobacco, while the black areas correspond to pixels classified as foreign materials. 

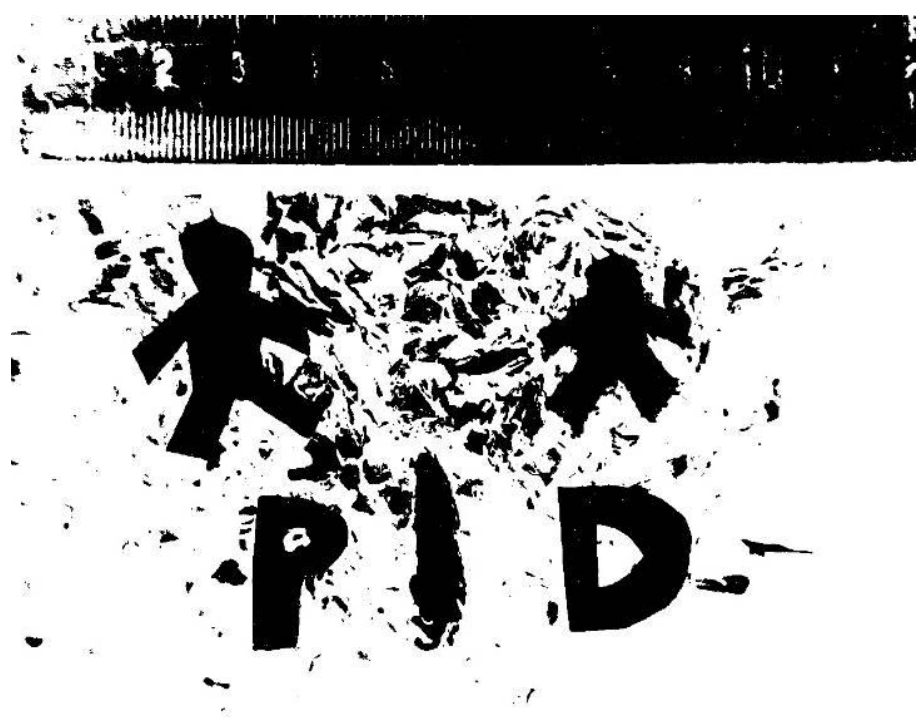

Fig. 7. Result of the discrimination phase using the SAM algorithm. White areas correspond with pixels detected as tobacco; black areas are identified as foreign objects.

Figure 7 shows two of the problems encounter during the experiments. First, the presence of shadows, resulting in zones for which no discrimination is possible. However, this can be easily solved with a better implementation of the light source, using more LEDs and a different spatial distribution of the diodes.

The second problem is related to the occurrence of false positives (detection of a non-existent foreign object), that should be minimized. With high resolution images, many single pixels can be incorrectly detected as foreign object mainly due to shadows, discrimination errors or dead pixels of the camera. In a real application, this will trigger a mechanical system that will throw away a large quantity of tobacco leaves around the (incorrectly detected) foreign object. To reduce this problem, a post-processing algorithm known as k-NN (k-Nearest Neighbours) [15] has been implemented. This algorithm is able to modify the classification of a pixel based on the number of similar pixels in the neighborhood. This approach has a spatial low-pass effect on the result, and foreign objects smaller than a number of pixels are not detected. This minimum size can however be adjusted by means of the size of the processing windows of the k-NN algorithm. Figure 8 shows the classification result after a k-NN post-processing with a $41 \mathrm{x} 41$ pixels window (corresponding to object sizes of about $4 \times 4 \mathrm{~mm})$. 


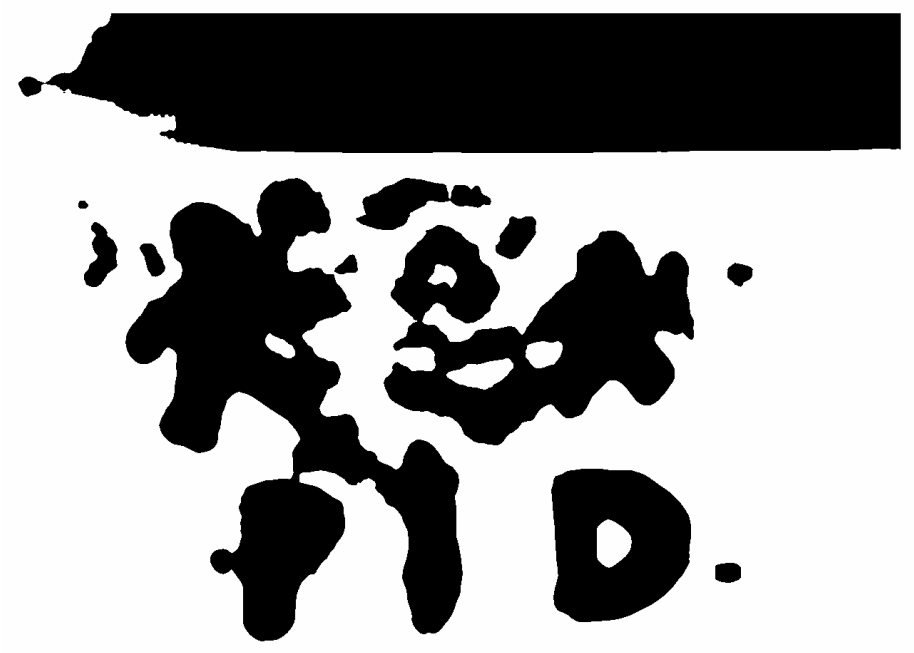

Figure 8. Result of the discrimination phase after a post-processing with a k-NN algorithm with $41 \times 41$ pixels window. Most of the false positives have been removed.

The same experimental setup and processing scheme has been applied to the image shown in Figure 9. This is a set of foreign objects (threads of rope, leather, plastic wrapping and a dry leave of a non-tobacco tree) over chopped tobacco leaves (tobacco is only seen in the upper part and the bottom-left corner of the image). This particular arrangement is more difficult to classify because the color of the objects is quite the same of the tobacco leaves, in particular, the tree leave (Platanus hispanica. ex Munchh.).

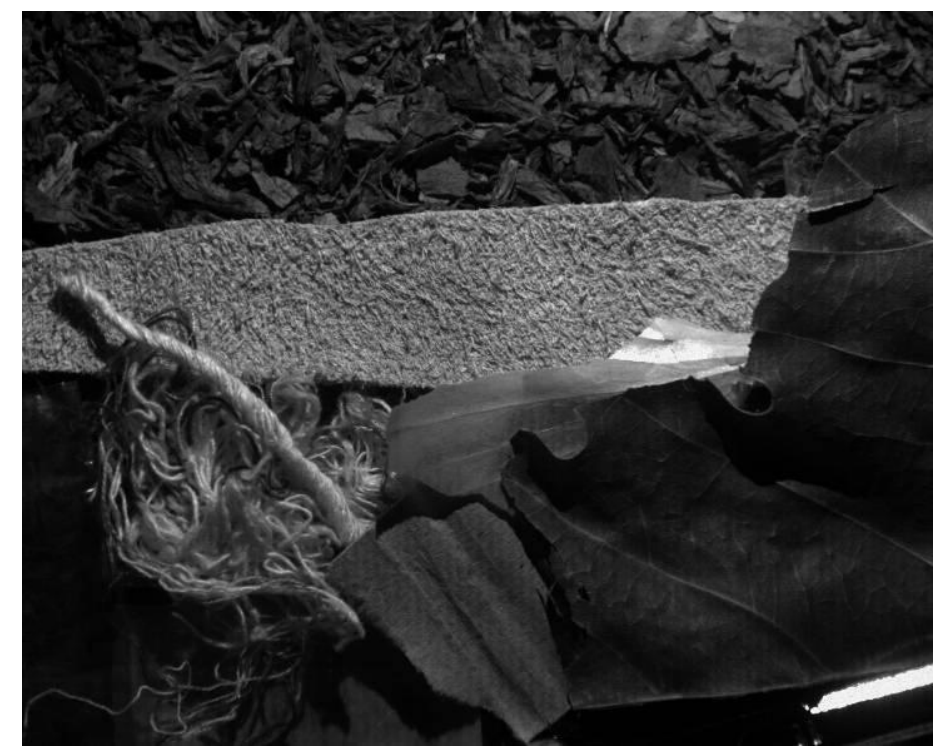

Figure 9. Another arrangement of foreign objects and tobacco leaves.

The result of the classification is shown in Figure 10, without (left) and with (right) k-NN processing. It can be seen that after k-NN processing, the classification is mostly correct, with the exception of part of the tree leave. Even with the misclassification of this fragment of the tree leave, this result is considered relevant because conventional color imaging 
would not be able to discriminate both kinds of vegetal materials due to the almost equal color of tobacco and nontobacco dry leaves.
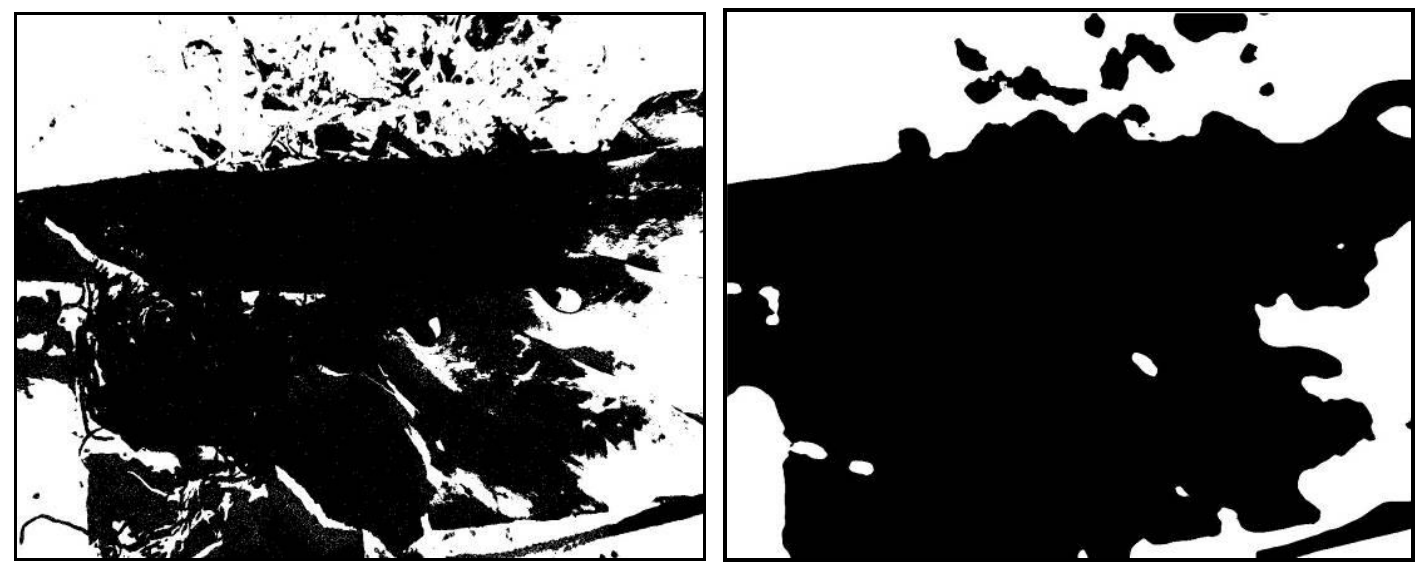

Figure 10. Result of the discrimination without (left) and with (right) k-NN post-processing.

\section{DISCUSSION}

A multispectral discrimination system based on an adaptive light source has been presented in this paper. The light source is based on a set of LEDs which are used to illuminate the material under study in a sequential way. The wavelengths of the LEDs, which are the most relevant for the discrimination process, are chosen in advance, through the SFFS dimensionality reduction algorithm. The spectral planes captured at each wavelength are processed with SAM algorithm, which offers a classification for every pixel in the images based on its spectral features.

A prototype of the system has been implemented and tested for a particular application: the automatic on-line detection of foreign objects among chopped tobacco leaves, a current problem in the tobacco industry. The results show the ability of this approach to discriminate between tobacco leaves and typical foreign objects, like candy wrappers, threads of cotton, wrapping paper, etc.

However, some aspects need to be improved. First, the occurrence of shadows in the image should be minimized, as they produce wrong classification of the materials in the image. A better implementation of the light source, with more LEDs and a larger emitting area should solve this problem. The discrimination of materials with almost equal spectral features (for example, tobacco and non-tobacco dry leaves) should also be improved. More research effort is being focused at the moment in these two particular aspects: a better implementation of the light source, and over an improved processing algorithm to reduce the classification error.

Acknowledgments: The authors would like to thank to the projects Spanish TEC'2005-08218-C02-02 and TEC'200767987-C02-01 for their financial support.

\section{REFERENCES}

[1] Duncan, D.B., Leeson, G., “Cost-effective real-time multispectral digital video imaging”, Proc. SPIE, 3649, 100-108 (1999).

[2] Xing J., Bravo C, Jancsók P.T., Ramon H. and Baerdemaeker J., "Detecting Bruises on 'Golden Delicious' Apples using Hyperspectral Imaging with Multiple Wavebands,” Biosystems Engineering, 90, $27-36$ (2005).

[3] Chang, C-I., [Hyperspectral Data Exploitation, Theory and Applications], John Wiley \& Sons, New Jersey, (2007).

[4] Vaarala, T., Aikio, M. and Keraenen, H., "An advanced prism-grating-prism imaging spectrograph in on-line industrial applications", Proc. SPIE, 3101, 322-330 (1997). 
[5] Conde, O.M., Amado, M. García-Allende, P.B., Cobo, A. and López-Higuera, J.M., "Evaluation of PCA dimensionality reduction techniques in imaging spectroscopy for foreign object detection," Proc. SPIE, 6565, 65650M (2007).

[6] Fukunaga, K., [Statistical Pattern Recognition], Academic Press, $2^{\text {nd }}$ edition, California \& London, $399-440$ (1990).

[7] Gomez-Chova, L., Calpe, J., Camps-Valls, G., Martin, J.D., Soria, E., Vila, J., Alonso-Chorda, L. and Moreno, J., "Feature selection of hyperspectral data through local correlation and SFFS for crop classification", 2003 IEEE International Geoscience and Remote Sensing Symposium, Proceedings (IEEE Cat. No.03CH37477), 555-557 (2003).

[8] Ferri F.J., Pudil P., Hatef M. and Kittler J., "Comparative study of techniques for large-scale feature selection", Pattern Recognition in Practice IV, Multiple Paradigms, Comparative Studies and Hybrid Systems, eds. E. S. Gelsema and L. S. Kanal. Amsterdam: Elsevier, 403-413 (1994).

[9] García-Allende, P.B., Conde, O.M., Mirapeix, J., Cubillas, A.M. and López-Higuera, J.M., "Relevant Information Extraction from Hyperspectral Images for Quality Control Industrial Processes," ODIMAP V Int. Symp. (5 ${ }^{\text {th }}$ Topical Meeting on Optoelectronic Distance/Displacement Measurements and Applications), Digest Tech. Papers , 271-276 (2006).

[10] Chen, C.H., [Fuzzy Logic and Neural Network Handbook], McGraw-Hill, New York, (1996).

[11] O'Farrell, M., Lewis, E., Lyons, W.B. and Jackman, N., "Combining principal component analysis with an artificial neural network to perform online quality assessment of food as it cooks in a large-scale industrial oven”, Sensors and Actuators B-Chemical, 107, 104 -112 (2005).

[12] Garcia-Allende, P.B., Conde, O.M., Cubillas, A.M., Jaúregui, C. and Lopez-Higuera, J.M., "New raw material discrimination system based on a spatial optical spectroscopy technique," Sensors and Actuators A-Physical, 135, 605-612 (2007).

[13] Kruse, F. A., Lefkoff, A. B., Boardman, J. W., Heiedbrecht, Shapiro, A. T., Barloon, P. J. and Goetz, A. F. H., “The Spectral Image Processing System (SIPS) - Software for Integrated Analysis of AVIRIS Data," Summaries of the 4th Annual JPL Airborne Geoscience Workshop, JPL Pub-92-14, AVIRIS Workshop. Jet Propulsion Laboratory, Pasadena, CA, 23-25 (1992).

[14] Garcia-Allende, P.B., Conde, O.M.; Mirapeix, J., Cubillas, A.M. and Lopez-Higuera, J.M., "Data processing method applying Principal Component Analysis and Spectral Angle Mapper for imaging spectroscopic sensors," Proc. SPIE, 6619, 66193Q (2007).

[15] Fukunaga, K., [Statistical Pattern Recognition], Academic Press, $2^{\text {nd }}$ edition, California \& London, 300-364 (1990). 